CORRECTION

https://doi.org/10.1038/s41586-019-1328-7

\title{
Author Correction: Neutrophils \\ support lung colonization of metastasis-initiating breast cancer cells
}

Stefanie K. Wculek \& Ilaria Malanchi

Correction to: Nature https://doi.org/10.1038/nature16140,

published online 09 December 2015.

In this Letter, the Supplementary Information file was resupplied without the final two pages, which included two tables-one that listed primers and one that listed antibodies. These two tables have now been added to the Supplementary Information to this Amendment. The original Letter has not been corrected.

Supplementary Information is available in the online version of this Amendment. 